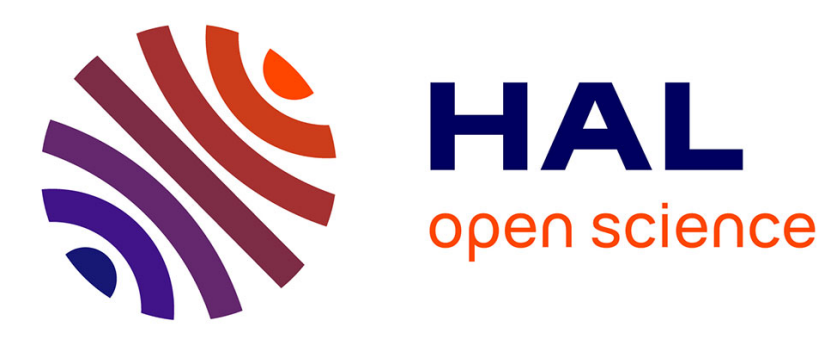

\title{
Effect of tube heat conduction on the pulsating heat pipe start-up
}

Iaroslav Nekrashevych, Vadim Nikolayev

\section{To cite this version:}

Iaroslav Nekrashevych, Vadim Nikolayev. Effect of tube heat conduction on the pulsating heat pipe start-up. Applied Thermal Engineering, 2017, 117, pp.24 - 29. 10.1016/j.applthermaleng.2017.02.013 . cea-01760895

\section{HAL Id: cea-01760895 https://hal-cea.archives-ouvertes.fr/cea-01760895}

Submitted on 9 Apr 2018

HAL is a multi-disciplinary open access archive for the deposit and dissemination of scientific research documents, whether they are published or not. The documents may come from teaching and research institutions in France or abroad, or from public or private research centers.
L'archive ouverte pluridisciplinaire HAL, est destinée au dépôt et à la diffusion de documents scientifiques de niveau recherche, publiés ou non, émanant des établissements d'enseignement et de recherche français ou étrangers, des laboratoires publics ou privés. 


\title{
Effect of tube heat conduction on the pulsating heat pipe start-up
}

\author{
Iaroslav Nekrashevych ${ }^{1}$, Vadim S. Nikolayev* \\ Service de Physique de l'Etat Condensé, CEA, CNRS, Université Paris-Saclay, CEA Saclay, 91191 Gif-sur-Yvette Cedex, France
}

\begin{abstract}
In this work, we present our first simulation results on the start-up, functioning and stopping (dry-out) of the multi-branch pulsating heat pipe (PHP) accounting for the fluid-tube thermal interaction and bubble generation (boiling). A theoretical model that generalizes an earlier proposed approach is described. It is shown that the account of tube heat conduction changes substantially the simulated PHP behavior. In particular, in the presence of tube heat conduction, the simulated PHP cannot provide stable oscillations without bubble generation. While the bubble generation may not be directly involved in the development of first oscillations, its role is crucial in preventing the oscillations halt. The mechanism of the oscillation sustainment by bubble generation is discussed. The PHP simulation shows basic phenomena of bubble interaction and regimes observed experimentally in transparent PHPs. The PHP ceases functioning when the evaporator power is larger than a threshold. The liquid films are evaporated so the evaporator dries out completely and the oscillations stop; the evaporator temperature rises steeply.
\end{abstract}

Keywords: pulsating heat pipe, oscillation, liquid films, phase change, simulation

\section{Introduction}

Pulsating (or oscillating) heat pipe (PHP) is a simple capillary tube bent into branches meandering between hot and cold spots and partially filled with a two-phase, usually single component working fluid. During PHP functioning, a moving pattern of multiple vapor bubbles separated by liquid plugs forms spontaneously inside the tube. Because of their simplicity and high performance, PHP's are often considered as highly promising [1]. Their industrial application is however limited because the functioning of PHPs is not completely understood; the absence of calculation tools that would allow their dimensioning is a substantial obstacle to their development.

During the last decade, researchers have extensively studied PHP $[2,3]$. It has been observed by many researchers that the main flow pattern inside the PHP is the slug flow, i.e. the flow of the "Taylor bubbles" where the gas is surrounded by liquid films. A major part of mass exchange occurs on their interface with the vapor like in the conventional heat pipes. Since the mass exchange provides both a moving force for the oscillations and the heat exchange, the films are extremely important for the PHP functioning.

Because of complex bubble and plug interactions and nonstationary dynamics, correlation-based system level approaches fail to predict the heat transfer in multi branch PHP and its direct numerical simulation seems to be the only possibility. One dimensional (1D) simulation models is the best choice because they are the simplest and at the same time are capable to de-

\footnotetext{
*Corresponding author: vadim.nikolayev@cea.fr, Ph.: +33169089488

${ }^{1}$ On leave from Heat Pipe Laboratory, Heat and Power Engineering Faculty, National Technical University of Ukraine "Kyiv Polytechnic Institute", Kyiv, 03056, Ukraine
}

scribe the relevant physical phenomena. The pioneering approach [4] introduced basic principles for the 1D modeling. The PHP meander was represented by a straight tube with periodic boundary conditions; the evaporator, adiabatic and condenser sections followed each other sequentially. A coherent thermodynamic description of vapor has been introduced. The vapor was described as a compressible ideal gas which allowed its spring-like action. The heat conduction in the liquid plugs has been introduced. The imposed fixed temperatures $T_{c}, T_{e}$ at the internal tube walls were assumed. Periodic oscillations were encountered. However, their amplitude was weak (smaller than the evaporator size), which contradicts the experimentally observed behavior.

One knows that the strongest heat and mass exchange occurs from thin liquid films. The films with uniform thickness $\delta_{f}$, but varying length were introduced into the modeling [5] of the simplest, single branch PHP so that a partial evaporator dry-out could be described. The introduction of the heat conduction inside the tube walls [6] enabled the fluid-solid thermal interaction. The latter is quite important because it accounts for such effects as e.g. transfer of the heat from hot liquid plugs to the walls, heat accumulation in the solid and its later reinjection into the fluid. Besides, much realistic imposed heat power thermal boundary conditions become possible to simulate. The approach [6] has been reused with some improvements by another group [7] without correcting the crucial default in the model [6]: unlike [4], the vapor heat exchange was described with an inconsistent equation (cf. [8]), without which it was apparently impossible to obtain large amplitude oscillations. The vapor phase modeling problem has been analyzed and a new, "film evaporation/condensation model" (FEC) has been developed [9] for the single branch PHP. The FEC model is deprived 


\begin{tabular}{|ll|}
\hline Nomenclature & $\lambda$ heat conductivity $[\mathrm{W} /(\mathrm{m} \mathrm{K})]$ \\
$c$ specific heat at constant pressure $[\mathrm{J} /(\mathrm{kg} \mathrm{K})]$ & $\phi$ volume fraction of liquid in PHP \\
$D$ heat diffusivity $\left[\mathrm{m}^{2} / \mathrm{s}\right]$ & $\rho$ density $\left[\mathrm{kg} / \mathrm{m}^{3}\right]$ \\
$d$ tube inner diameter $[\mathrm{m}]$ & Superscripts \\
$h_{l v}$ latent heat $[\mathrm{J} / \mathrm{kg}]$ & $l$ left \\
$L$ length $[\mathrm{m}]$ & $r$ right \\
$M$ total number of bubbles or plugs & Subscripts \\
$m$ mass of vapor $[\mathrm{kg}]$ & $c$ condenser \\
$N$ total number & $e$ evaporator \\
$N u$ Nusselt number & $f$ liquid film \\
$P$ power $[\mathrm{W}]$ & $f b$ feed back section $(\mathrm{vertical} \mathrm{in} \mathrm{Fig.} 1)$ \\
$p$ pressure; $p_{i}:$ in the bubble $i[\mathrm{~Pa}]$ & $i$ bubble or plug identifier \\
$q$ heat flux $\left[\mathrm{W} / \mathrm{m}^{2}\right]$ & $l$ liquid \\
$S$ cross-section area $\left[\mathrm{m}^{2}\right]$ & $m$ meniscus \\
$T$ temperature $\left[T_{i}:\right.$ of vapor] $[\mathrm{K}]$ & $n e x t$ next bubble \\
$t$ time $[\mathrm{s}]$ & $n u c l$ nucleation \\
$U$ heat transfer coefficient $\left[\mathrm{W} /\left(\mathrm{m}^{2} \mathrm{~K}\right)\right]$ & $p$ PHP spatial period \\
$x, X$ abscissa measured along the PHP tube $[\mathrm{m}]$ & sat at saturation \\
$G r e e k$ symbols & $t$ total \\
$\Delta$ difference & $t h r$ threshold \\
$\delta$ thickness $[\mathrm{m}]$ & $v$ vapor \\
\hline
\end{tabular}

of the above mentioned shortcomings and truthfully reproduces the experimental behavior both qualitatively and quantitatively, at least for the single branch PHP [9-11]. The FEC model has been generalized later to the multi-branch PHP [12] with the imposed temperatures $T_{c}, T_{e}$ of the internal tube walls. An alternative film description where the film is divided by the equal length pieces of variable thickness has been introduced [13]. Within such an approach, it is also possible to describe the evaporator dry-out [14]. The bubble generation has been introduced by the same group, which is an important step forward. Indeed, such an approach is much heavier computationally as one needs to in addition to liquid plugs, discretize the liquid films. Probably for this reason, the wall temperature was assumed to be constant. The oscillation amplitude was small with respect to the evaporator size.

The main issue in the multi branch PHP is the oscillation start-up that one needs to master to develop reliable PHP for industrial needs. Because of multitude of parameters that may be relevant, it is a difficult task. For this reason, we began the start-up study with applying the FEC model to the simplest, single branch PHP $[15,16]$. First, the start-up has been studied for the stepwise variation of the temperature $T_{w}$ of the internal tube wall (simply "wall" in the following for brevity) from $T_{c}$ to $T_{e}$ [15]. It has been found that the dimensionless control parameters for the start-up depended crucially on $\delta_{f}$. The start-up in the presence of the tube heat conduction and the fluid-wall heat exchange has been considered in the subsequent work [16]. A constant homogeneously distributed heat power $P_{e}$ applied to the evaporator has been simulated while the wall temperature $T_{c}$ in the condenser was fixed. It has been found that the nonzero tube conduction completely changes the start-up criteria. In particular, they become independent of the liquid film pa- rameters. It has been shown that the tube thermal properties considerably influence the single branch PHP start-up.

The main problem of the published up to now PHP modeling results is that they fail to describe even qualitatively the usual bubble patterns observed experimentally. This paper presents our first results of multibranch PHP simulation accounting for the fluid-tube thermal interaction and bubble generation (boiling) with the same boundary conditions as in Ref. [16]: fixed $P_{e}$ and $T_{c}$. The in-house CASCO software (French abbreviation for Code Avancé de Simulation du Caloduc Oscillant: advanced PHP simulation code, $\mathrm{cf}$. Fig. 1) has been used for simulations. The gravity is switched off; the discussed below simulations thus correspond to the horizontal PHP orientation.

\section{FEC model with tube heat conduction}

The simulation is based on the FEC model for the closed loop PHP described earlier [12]. The PHP meandering tube consisting of a sequence of different PHP sections is projected to a straight axis $x$. One PHP spatial unit of the length $L_{p}=$ $2 L_{a}+L_{c}+L_{e}$ is assumed to contain the sections in the following order: evaporator, adiabatic, condenser, and again adiabatic. The point $x=0$ coincides with the beginning of evaporator (Fig. 1). $L_{t}=N_{p} L_{p}+L_{f b}$ is the total PHP length, where $L_{f b}$ represents the length of the feedback section (shown vertical in Fig. 1). The periodic boundary conditions are imposed in such a way that the local values for all the variables at the points $x$ and $x+L_{t}$ are the same for any $x$; the train of bubbles moves along an infinite axis. Within such a description, the coordinate $X_{i}^{l}$ of the left meniscus of the bubble $i$ is always smaller than that of its right meniscus $X_{i}^{r}$; the bubble order does not change during their motion. The total number $M$ of bubble-plug couples may 


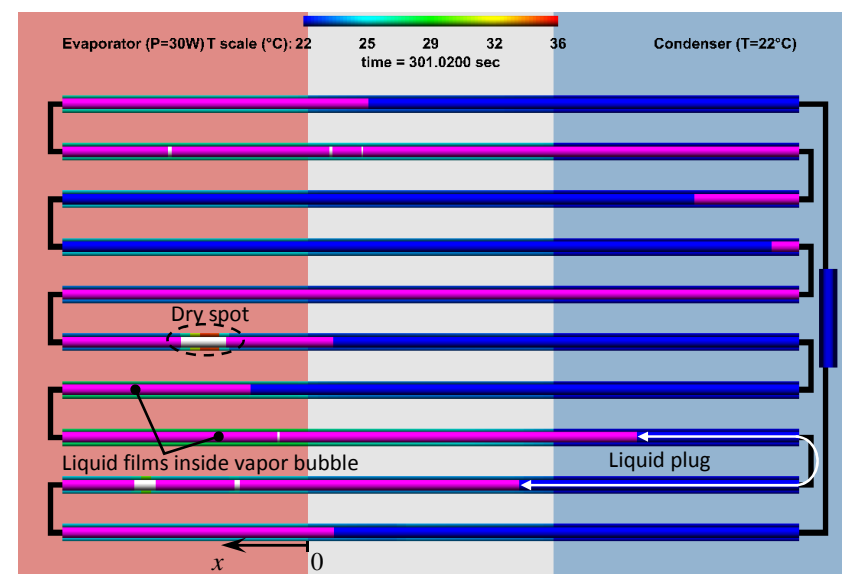

Figure 1: The PHP geometry pictured by the PHP_Viewer postprocessor of the CASCO software. Thin liquid films (in violet) cover the internal tube walls inside of the vapor bubbles, except of the dry spot areas (white). The liquid plugs are shown in blue. The wall temperature is shown with the color varying from blue to red; its scale is indicated at the top. The round turns are not pictured for simplicity; black lines just connect equivalent points which correspond to the extreme points of each turn.

change in time because of bubble generation, coalescence and disappearance. The liquid plug which is the right neighbor to the bubble $i$ is identified with the same index $i$. The left end of the plug is thus $X_{i}^{r}$ and the right is $X_{i, \text { next }}^{l}$, where $X_{i, \text { next }}^{l}=X_{i+1}^{l}$ unless $i=M$, in which case $X_{i, n e x t}^{l}=X_{1}^{l}+L_{t}$.

The following main assumptions are used:

1. Bubble and film (if any) occupy all the tube cross-section in the bubbles; liquid occupies all the tube within the liquid plugs (no dispersed flow).

2. The film is of the fixed thickness $\delta_{f}$ defined a posteriori by the average plug velocity during the established oscillations by using a formula conventional for the Taylor bubble film as described in [15].

3. The films are deposited by the receding meniscus and "eaten up" by the advancing meniscus.

4. Film evaporation leads to the reduction of the film area; the film condensation causes its increase unless it is impossible (e.g. no dry area in the bubble; cf. assumption 2 ). In the latter case the condensed mass is equally distributed between the neighboring liquid plugs to provide the fluid mass conservation.

5. The films may be partially or completely evaporated in the evaporator or adiabatic section. The film evaporation rate is proportional to the film area and to the local superheating.

6. There is evaporation from each meniscus. Its rate is proportional to the local superheating at the meniscus position.

7. Vapor obeys the ideal gas equation of state and may be overheated [10], liquid is incompressible.

8. Bubbles and plugs disappear when their length becomes smaller than a small threshold, $L_{t h r}$; a threshold mass $m_{t h r}$ is defined for a bubble.
9. Bubble of the length $L_{n u c l}$ is generated at any point $x$ inside the plug $i$ if the local superheating exceeds a nucleation barrier, $T_{w}(x)-T_{\text {sat }}\left(p_{l}\right)>\Delta T_{\text {nucl }}$, where $p_{l}=$ $p_{i}+\left(p_{i, \text { next }}-p_{i}\right)\left(x-X_{i}^{r}\right) /\left(X_{i, \text { next }}^{l}-X_{i}^{r}\right)$ is the local liquid pressure assumed to vary linearly along a plug. The nucleation barrier $\Delta T_{\text {nucl }}$ depends strongly on the surface properties. It is assumed to be constant.

Note that unlike earlier bubble generation approaches, there are no preferential nucleation sites in the model.

The general algorithm and the structure of the CASCO code have been already discussed [12]. We describe here only the features that have to be changed in order to implement the fluidwall heat exchange.

To simulate as precisely as possible the dry wall area under the conditions where $T_{w}$ varies in space, a notion of the effective evaporator needs to be introduced. It is a part of the wall within the bubble $i$ which is either dry (i.e. uncovered by the film) or along which $T_{w}$ exceeds $T_{\text {sat }}\left(p_{i}\right)$. In the latter case, the film might be currently continuous but will rupture next time moment. There may be several effective evaporators per bubble and, accordingly, several film pairs formed by the right and left films. The number of effective evaporators is denoted $N_{e, i}$. The left and right film edge positions (i.e. those of triple contact lines) are denoted with $X_{f, i}^{l, k}$ and $X_{f, i}^{r, k}$ for the $k$ th effective evaporator, cf. Fig. 2. The following inequalities $X_{i}^{l} \leq X_{e, i}^{l, 1} \leq$ $X_{f, i}^{l, 1} \leq X_{f, i}^{r, 1} \leq X_{e, i}^{r, 1} \leq \cdots \leq X_{e, i}^{l, N_{e, i}} \leq X_{f, i}^{l, N_{e, i}} \leq X_{f, i}^{r, N_{e, i}} \leq X_{e, i}^{r, N_{e, i}} \leq X_{i}^{r}$ are thus satisfied and should be enforced numerically to provide the correct film management. The film dynamics is described by the equations that interpret the assumptions 1-4,

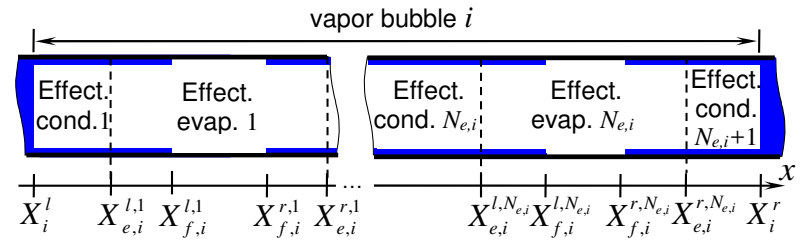

Figure 2: Geometry of the effective evaporators and condensers.

$$
\begin{aligned}
& \dot{X}_{f, i}^{l, k}=\left\{\begin{array}{l}
\dot{X}_{i}^{l}, \text { if } X_{f, i}^{l, k} \leq X_{i}^{l} \text { and } \dot{X}_{i}^{l} \geq 0 \\
-\frac{\dot{m}_{f c, i}^{k}}{2 \rho \pi d \delta_{f}}, \text { if } X_{e, i}^{l, k} \geq X_{f, i}^{l, k} \text { and } X_{f, i}^{l, k}<X_{f, i}^{r, k} \\
-\frac{\dot{m}_{f e, i}^{l, k}}{\rho \pi d \delta_{f}}, \text { otherwise. }
\end{array}\right. \\
& \dot{X}_{f, i}^{r, k}=\left\{\begin{array}{l}
\dot{X}_{i}^{r}, \text { if } X_{f, i}^{r, k} \geq X_{i}^{r} \text { and } \dot{X}_{i}^{r} \leq 0 \\
\frac{\dot{m}_{f c, i}^{k+1}}{2 \rho \pi d \delta_{f}}, \text { if } X_{e, i}^{r, k} \leq X_{f, i}^{r, k} \text { and } X_{f, i}^{l, k}<X_{f, i}^{r, k} \\
\frac{\dot{m}_{f e, i}^{r, k}}{\rho \pi d \delta_{f}}, \text { otherwise. }
\end{array}\right.
\end{aligned}
$$

where the dot means the time derivative. According to the assumption 5 , the vapor mass change of the $i$ th bubble caused by 
the evaporation of right and left films of the $k$ th effective evaporator are defined by the expressions

$$
\begin{aligned}
& \dot{m}_{f e, i}^{r, k}=\frac{U_{f} \pi d}{h_{l v}} \int_{X_{f, i}^{r, k}}^{X_{e, i}^{r, k}}\left(T_{w}(x)-T_{s a t, i}\right) \mathrm{d} x, \\
& \dot{m}_{f e, i}^{l, k}=\frac{U_{f} \pi d}{h_{l v}} \int_{X_{e, i}^{l, k}}^{X_{f, i}^{l, k}}\left(T_{w}(x)-T_{s a t, i}\right) \mathrm{d} x,
\end{aligned}
$$

where $T_{\text {sat }, i}=T_{\text {sat }}\left(P_{i}\right)$ and $U_{f} \sim \lambda_{l} / \delta_{f}$ within a coefficient of the order unity [9]. The vapor mass change of the $i$ th bubble caused by the condensation in the $k$ th effective condenser is

$$
\dot{m}_{f c, i}^{k}=\frac{U_{f} \pi d}{h_{l v}}\left\{\begin{array}{l}
\int_{X_{i}^{l}}^{X_{e, i}^{l, k}}\left(T_{w}(x)-T_{s a t, i}\right) \mathrm{d} x, k=1 \\
\int_{X_{e, i}^{r, k-1}}^{X_{e, i}^{l, k}}\left(T_{w}(x)-T_{s a t, i}\right) \mathrm{d} x, 1<k \leq N_{e, i} \\
\int_{X_{e, i}^{r, k-1}}^{X_{i}^{r}}\left(T_{w}(x)-T_{s a t, i}\right) \mathrm{d} x, k=N_{e, i}+1
\end{array}\right.
$$

According to the assumption 6 , the vapor mass change of the $i$ th bubble caused by the phase change at the meniscus (more precisely, at a meniscus part adjacent to the wall) is

$$
\dot{m}_{m, i}=\frac{U_{m} \pi d L_{m}}{h_{l v}}\left[\left(T_{w}\left(X_{i}^{l}\right)+T_{w}\left(X_{i}^{r}\right)-2 T_{s a t, i}\right]\right.
$$

Total mass change rate $\dot{m}_{i}$ for the $i$-th bubble:

$$
\dot{m}_{i}=\dot{m}_{m, i}+\dot{m}_{f c, i}^{N_{e, i}+1}+\sum_{k=1}^{N_{e, i}}\left(\dot{m}_{f e, i}^{l, k}+\dot{m}_{f e, i}^{r, k}+\dot{m}_{f c, i}^{k}\right) ;
$$

The temperature $T_{w}$ is determined from the equation [16]

$$
\frac{\partial T_{w}}{\partial t}=D_{w} \frac{\partial^{2} T_{w}}{\partial x^{2}}+\frac{j_{w}}{\rho_{w} c_{w}}
$$

solved within the evaporator and adiabatic section, where

$$
j_{w}=\frac{\pi}{S_{w}} \begin{cases}\frac{P_{e}}{N_{p} L_{e}}-q_{\text {fluid }} d & \text { if } x \in \text { evaporator, } \\ -q_{\text {fluid }} d & \text { if } x \in \text { adiab. sec., }\end{cases}
$$

where $S_{w}$ is the tube material cross-section area. The heat flux $q_{\text {fluid }}=U_{\text {fluid }}(x)\left[T_{w}(x)-T_{\text {fluid }}(x)\right]$ is transferred from the internal tube wall to the fluid, where

$$
T_{\text {fluid }}(x)= \begin{cases}T_{i}, & \text { if } x \in \text { dry area, } \\ T_{s a t, i}, & \text { if } x \in \text { film, } \\ T_{l}(x), & \text { if } x \in \text { liquid. }\end{cases}
$$

The heat exchange coefficient $U_{\text {fluid }}$ is either $U_{v}=N u_{v} \lambda_{v} / d$, $U_{f}$ or $U_{l}=N u_{l} \lambda_{l} / d$ for the respective regions; $N u_{v}=6$ [10], $N u_{l} \simeq 4$ characteristic for the laminar single phase flow. The temperature $T_{w}(x)=T_{c}$ within the condenser. The PHP material properties used in simulation are shown in Table 1. Initially, several equidistant bubbles are distributed along the PHP; $T_{w}(t=0)=T_{c}$.
Table 1: Parameters used for the simulation.

\begin{tabular}{ll}
\multicolumn{1}{c}{ Table 1: Parameters used for the simulation. } \\
\hline \hline Lengths of zones & $L_{e}=L_{a}=L_{c}=0.1 \mathrm{~m}$ \\
Number of turns & $N_{p}=5$ \\
Feedback length & $L_{f b}=2 \mathrm{~cm}$ \\
Filling ratio & $\phi=0.5$ \\
Inner diameter & $d=3 \mathrm{~mm}$ \\
Outer diameter & $4 \mathrm{~mm}$ \\
Condenser temperature & $T_{c}=22^{\circ} \mathrm{C}$ \\
Fluid & Water \\
Tube material & Hastelloy \\
Time step & $0.1 \mathrm{~ms}$ \\
Tube mesh size & $2 \mathrm{~mm}$ \\
Film thickness & $\delta_{f}=40 \mu \mathrm{m}$ \\
Threshold length & $L_{t h r}=10 \mu \mathrm{m}$ \\
Nucleated bubble length & $L_{n u c l}=300 \mu \mathrm{m}$ \\
\hline \hline
\end{tabular}

\section{Simulation results}

One may distinguish different regimes of PHP functioning: start-up, steady oscillations, dry-out. It is well known that there are many internal events occurring in the PHPs. They are reproduced by simulation as described below. CASCO provides management of the following events occurring with vapor bubbles and liquid plugs: bubble generation, disappearance of bubbles and plugs, dry spot growth and disappearance. The bubble disappearance occurs according to the assumption 8 when a liquid plug overtakes another plug. The vapor pressure grows and fast condensation occurs. A composite long plug forms. Its mass and velocity is determined from the mass and momentum conservation [12]. The bubble is deleted together with all variables associated with it; the current time step is recalculated with a smaller number of equations. Bubbles disappear mainly in the condenser section.

A small plug may also disappear, in two cases. (i) When the plug moves over a dry area, it may spend all its liquid for the film deposition. (ii) When it remains still and there is intense evaporation on its meniscus. However such an event is much less likely.

A typical PHP start-up and functioning is illustrated by Fig.3. The average temperature remains stable within $\pm 1{ }^{\circ} \mathrm{C}$ during a long period of time. An insert shows that the start-up lasts less than $5 \mathrm{~s}$.

One of the main results of this study is the absence of the multibranch PHP start-up without bubble generation. Whatever is $P_{e}$, the oscillation starts initially but chain bubble coalescence occurs and, eventually, all the liquid gathers in a single plug; its oscillations decline quickly. This seems to be an effect of the tube heat conduction. When the wall temperature was imposed and varied stepwise from condenser to evaporator [12], this chain bubble coalescence was observed for a heating power larger than a threshold. However the PHP start-up was possible in the absence of bubble generation for some temperature differences. The start-up of the single branch PHP is also possible in the heat conducting tube in the absence of bubble generation [16] probably because the average pressure inside PHP is imposed: the single branch PHP has an open end. 


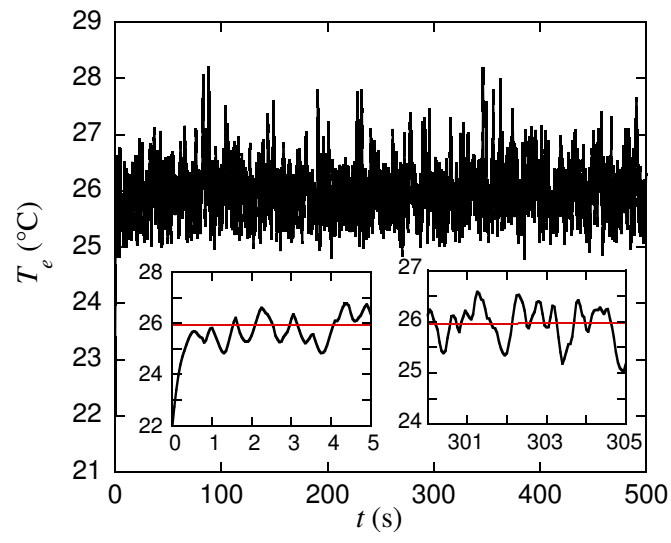

Figure 3: Time evolution of the averaged over evaporator temperature $T_{e}$. The time averaged $T_{e}$ value $\left\langle T_{e}\right\rangle=25.8^{\circ} \mathrm{C}$ is shown in red in the inserts. The PHP thermal resistance calculated with it is $0.14 \mathrm{~K} / \mathrm{W}$.

\subsection{Start-up and bubble generation}

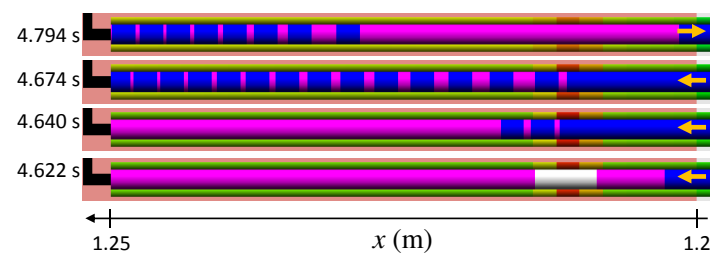

Figure 4: An example of multiple bubble generation in the evaporator of the 9-th branch (2nd from the top in Fig. 1 corresponding to $1.2 \mathrm{~m}<x<1.25 \mathrm{~m}$ ) at $P_{e}=60 \mathrm{~W}$. The color significance is the same as in Fig. 1. Arrows show the direction of motion of the rightmost liquid plug.

When the bubble generation criterion (assumption 9) is satisfied, the bubble generation starts. If the wall is hot enough, many bubbles may be generated one after another at the same hot spot so that "bubble trains" are formed, cf. Fig.4 where the hot spot situates at $x \simeq 1.21 \mathrm{~m}$. This event can be seen in Fig. 5 where the time evolution of the left and right ends $\left(X_{l}\right.$, $X_{r}$ ) of many bubbles is shown for the same case as in Fig.4. For any particular newly born bubble, one can easily identify the left and right bubble ends in Fig. 5 because they form a divergent cusp point at the birth moment (encircled in red). As mentioned before, $X_{l}^{i} \leq X_{r}^{i}$ so the vertical distance between $X_{r}^{i}$ and $X_{l}^{i}$ curves defines the variable in time bubble length. The plug length (the distance between $X_{l}^{i+1}$ and $X_{r}^{i}$ ) can vary because of the meniscus phase change and because of the liquid film deposition by the receding plug meniscus if the other end advances along the dry wall.

Bubble birth and growth consume the latent heat so the bubble generating hot spot eventually cools down and the bubble generation ends. This is visible in Fig. 5. Right after generation, the velocity of the bubble train coincides with that of the parent plug. After the birth, the bubbles grow in the evaporator. The growth of a bubble in evaporator causes compression of the neighboring bubbles and the direction of plug motion can be inverted (cf. Fig.4 for $t=4.794 \mathrm{~s}$ ). This inversion can be also seen in Fig. 5 where the slope of curves (defining the plug velocity) changes its sign. Such an inversion means an oscilla-

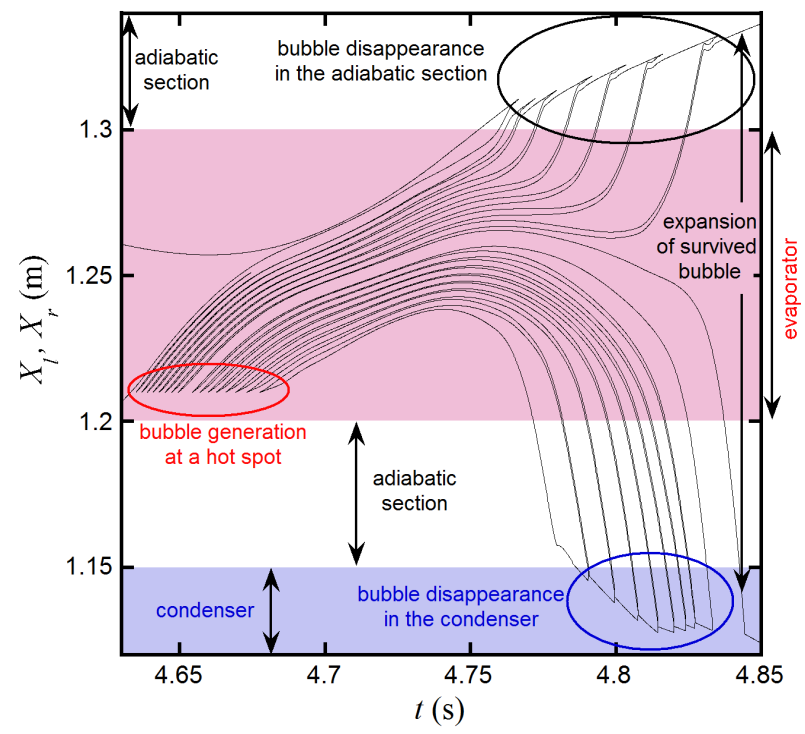

Figure 5: Time evolution of bubble end coordinates in the 9th and 10th branches. Each pair of solid lines corresponds to a bubble, i. e. to the temporal variation of its $X_{l}$ and $X_{r}$. Each starting (i.e. opening to the right) cusp point corresponds to the bubble generation at a hot spot. Each ending (i.e. opening to the left) cusp point corresponds to the bubble disappearance.

tion. This shows the mechanism of oscillation sustainment by bubble generation.

Because of the pressure rise discussed above, some of newly created bubbles may disappear (recondense) soon after creation once they reach a cooler condenser or adiabatic section. The bubble disappearance can be identified in Fig. 5 by the lines converging in a cusp (encircled in blue in condenser and in black in adiabatic section).

\subsection{PHP dry-out}

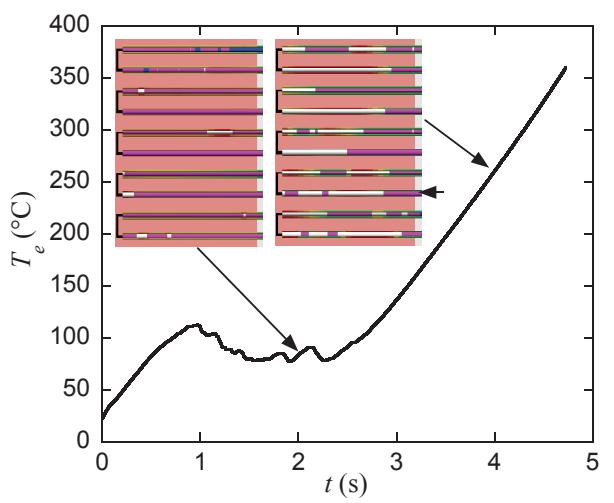

Figure 6: Tube wall temperature for $P_{e}=360 \mathrm{~W}$ averaged over evaporator: dry-out dynamics. The images of evaporator are shown for two different times $(t=2$ and $4 \mathrm{~s})$ to illustrate the dry area growth dynamics.

The accurate description of dry spots allows us to simulate the complete PHP halt by dry-out that occurs when $P_{e}$ exceeds a threshold. At a larger $P_{e}$, after a short transient, film length begins to decrease in the evaporator and large dry spots (white areas in the evaporator shown in Fig.6) grow. Within the dry 


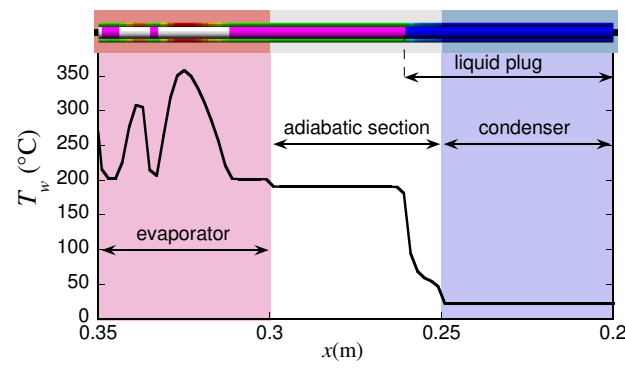

Figure 7: Temperature distribution along the third PHP branch indicated by an arrow in Fig. 6 for $P_{e}=360 \mathrm{~W}, t=4 \mathrm{~s}$. The same image is enlarged above the curve to indicate the position of film-covered (violet), liquid plug-covered (blue) and dry (white) areas.

spots, the heat transfer is much smaller than within the filmcovered area (because of the smaller vapor heat conductivity). High thermal gradients form along the tube (Fig.7) between the dry and film-covered areas in the evaporator. The fast film evaporation in evaporator leads to the pressure rise in all the bubbles located there. This means that all the liquid plugs are expelled almost simultaneously from the evaporator. Such an event causes strong compression of bubbles that situate in condenser and adiabatic sections. The vapor inside these bubbles condense until their complete disappearance. As a result, all the liquid gathers in the condenser while all the vapor is located in the evaporator. The pressure imbalance between the branches becomes weak which causes a decrease of oscillation amplitude. The evaporator temperature grows sharply (cf. Fig.6, after $2.5 \mathrm{~s}$ ) which makes the film disappearance irreversible.

The dry-out has been already simulated in a different dynamic film model [14]. However, the authors could not obtain the dry-out with bubble generation. In the present work the dry-out has been obtained with bubble generation, in agreement with numerous PHP experiments where bubble generation is always present.

\section{Conclusions}

We discuss here a theoretical model and numerical simulations of multi-branch PHPs in horizontal orientation (with no gravity influence) including the effects of heat conduction by the tube walls, fluid-tube thermal coupling and bubble generation. It has been found that in the presence of tube heat conduction the PHP start-up is impossible without the bubble generation. The oscillations may start because of initial deviation from equilibrium but they disappear soon because all liquid plugs coalesce. This behavior is different from the earlier studied case of the imposed evaporator and condenser temperatures with no tube heat conduction where the PHP start-up was possible [12]. In the presence of bubble generation, the oscillations of large amplitude are stable at long times. A mechanism of the oscillation sustainment by the bubble generation is revealed.

The present simulations can describe the PHP dryout. When the heating power is larger than a threshold, the stable oscillation do not start-up. The liquid films disappear in the evaporator and its temperature rises steeply; the oscillations stop.
Our simulations reproduce at least qualitatively many physical phenomena observed experimentally in transparent PHPs. One needs to perform more detailed studies to make comparisons with experimental data.

\section{ACKNOWLEDGMENTS}

The financial contributions of ANR in the framework of the project AARDECO ANR-12-VPTT-005-02 and of ESA within MAP INWIP are acknowledged. This work was presented at 18th Int. Heat Pipe Conf. and 12th Int. Heat Pipe Symp., Jeju, South Korea, June 12-16, 2016.

\section{References}

[1] S. Kim, Y. Zhang, J. Choi, Effects of fluctuations of heating and cooling section temperatures on performance of a pulsating heat pipe, Appl. Therm. Eng. 58 (1-2) (2013) 42 - 51, doi:10.1016/j.applthermaleng.2013.03.037.

[2] Y. Zhang, A. Faghri, Advances and Unsolved Issues in Pulsating Heat Pipes, Heat Transfer Eng. 29 (1) (2008) 20 - 44, doi:10.1080/01457630701677114.

[3] S. Khandekar, P. K. Panigrahi, F. Lefèvre, J. Bonjour, Local hydrodynamics of flow in a pulsating heat pipe: a review, Frontiers in Heat Pipes 1 (2) (2010) 023003, doi:10.5098/fhp.v1.2.3003.

[4] M. B. Shafii, A. Faghri, Y. Zhang, Thermal Modeling of Unlooped and Looped Pulsating Heat Pipes, J. Heat Transfer 123 (6) (2001) 1159 1172, doi:10.1115/1.1409266.

[5] R. T. Dobson, Theoretical and experimental modelling of an open oscillatory heat pipe including gravity, Int. J. Therm. Sci. 43 (2) (2004) 113 119, doi:10.1016/j.ijthermalsci.2003.05.003.

[6] B. Holley, A. Faghri, Analysis of pulsating heat pipe with capillary wick and varying channel diameter, Int. J. Heat Mass Transfer 48 (13) (2005) 2635 - 2651, doi:10.1016/j.ijheatmasstransfer.2005.01.013.

[7] M. Mameli, M. Marengo, S. Zinna, Numerical model of a multi-turn Closed Loop Pulsating Heat Pipe: Effects of the local pressure losses due to meanderings, Int. J. Heat Mass Transfer 55 (4) (2012) 1036 - 1047 , doi:10.1016/j.ijheatmasstransfer.2011.10.006.

[8] V. S. Nikolayev, Comment on "Flow and heat transfer of liquid plug and neighboring vapor slugs in a pulsating heat pipe" by Yuan, Qu, \& Ma, Int. J. Heat Mass Transfer 54 (9-10) (2011) 2226 - 2227, doi:10.1016/j.ijheatmasstransfer.2011.01.007.

[9] S. P. Das, V. S. Nikolayev, F. Lefèvre, B. Pottier, S. Khandekar, J. Bonjour, Thermally induced two-phase oscillating flow inside a capillary tube, Int. J. Heat Mass Transfer 53 (19-20) (2010) 3905 - 3913, doi:10.1016/j.ijheatmasstransfer.2010.05.009.

[10] P. Gully, F. Bonnet, V. Nikolayev, N. Luchier, T. Q. Tran, Evaluation of the vapor thermodynamic state in PHP, in: Proc. 17th Int. Heat Pipe Conf., Begell, ISBN 978-1-56700-453-3, 369 - 376, 2015.

[11] M. Rao, F. Lefèvre, S. Khandekar, J. Bonjour, Understanding transport mechanism of a self-sustained thermally driven oscillating two-phase system in a capillary tube, Int. J. Heat Mass Transfer 65 (2013) 451 - 459, doi:10.1016/j.ijheatmasstransfer.2013.05.067.

[12] V. S. Nikolayev, A Dynamic Film Model of the Pulsating Heat Pipe, J. Heat Transfer 133 (8) (2011) 081504, doi:10.1115/1.4003759.

[13] R. Senjaya, T. Inoue, Oscillating heat pipe simulation considering bubble generation. Part I: Presentation of the model and effects of a bubble generation, Int. J. Heat Mass Transfer 60 (0) (2013) 816 - 824, doi:10.1016/j.ijheatmasstransfer.2013.01.059.

[14] R. Senjaya, T. Inoue, Oscillating heat pipe simulation considering dryout phenomena, Heat Mass Transfer 50 (10) (2014) 1429-1441, doi:10.1007/s00231-014-1354-9.

[15] V. S. Nikolayev, Oscillatory instability of the gas-liquid meniscus in a capillary under the imposed temperature difference, Int. J. Heat Mass Transfer 64 (2013) 313 - 321, doi:10.1016/j.ijheatmasstransfer.2013.04.043. 
[16] V. S. Nikolayev, Effect of tube heat conduction on the single branch pulsating heat pipe start-up, Int. J. Heat Mass Transfer 95 (2016) 477 - 487, doi:10.1016/j.ijheatmasstransfer.2015.12.016. 